\title{
Research on the Translation Strategies of the Names of Traditional Chinese Medicine Classical Works under the Network Background Yan $\mathrm{Lu}^{1, \mathrm{a}}$
}

\author{
${ }^{1}$ School of Foreign Language, Shaanxi University of Chinese Medicine, Xianyang, Shaanxi 712046, \\ China
}

a13891831683@163.com

Keywords: Network background; names; traditional Chinese medicine; Classical works; translation; strategies

\begin{abstract}
Due to the rapid development of modern information technology, the international level of science and technology and cultural exchanges are more and more frequent. The translation of traditional Chinese Medicine classical works has become the key media for Chinese scholars to communicate with scholars in different countries and cultures. Due to the characteristics of traditional Chinese medicine, the translator's knowledge reserve and ability, the differences in cultural background and living habits of different countries and nations, there are good and bad translations in the translation works of TCM classics. In order to improve the translation quality of TCM classics and promote the internationalization of traditional Chinese medicine, this paper explores the translation skills of TCM classical works from the perspective of translation strategies of classical Chinese translation works under the network background.
\end{abstract}

\section{Introduction}

The Chinese nation has a long history, and Chinese medicine has a long history. It is the precious wisdom crystallization that we first summed up in the process of struggling with diseases. Although the mainstream medical and biological application technology research of Western medicine in modern society, but since the $19^{\text {th }}$ Century there is a growing number of western scholars who believe that traditional Chinese medicine has obvious advantages and unique value in the treatment and prevention of diseases. With the rise and influence of the concept of "return" in the world, Chinese medicine has received more and more attention and recognition from the international community [1-3]. Since the founding of the people's Republic of China, with the continuous efforts of Chinese scholars, the translation of traditional Chinese medicine books has made great progress, and it has been fruitful. Due to the characteristics of traditional Chinese medicine, the translator's knowledge reserve and ability, the differences in cultural background and living habits of different countries and nations, there are good and bad translations in the translation works of TCM classics $[4,5]$. In order to improve the translation quality of TCM classics and promote the internationalization of traditional Chinese medicine, this paper explores the translation skills of TCM classics from the perspective of translation strategies of classical Chinese translation works under the network background.

\section{Characteristics of TCM language}

Traditional Chinese medicine (TCM) is a broad and profound learning, which contains infinite wisdom. With the deepening and expansion of foreign exchange in traditional Chinese medicine, frequent translation of traditional Chinese medicine has become an inevitable trend of development. In recent years, many Chinese medicine scholars and translators have put forward their own views on the translation of traditional Chinese medicine, which plays a certain role in promoting the development of translation of traditional Chinese medicine [6]. But to achieve the standardization of Chinese medicine translation, we must start from the understanding of the characteristics of Chinese medicine language. The characteristics of Chinese language are as follows: 
(1)Take the image class

It is a kind of traditional Chinese thinking mode, which is based on the intuition and experience of the specific "image" to describe and analogical abstract things, to help people understand the world and understand the abstract things a basic Ideas and methods. China's ancient medical practitioners with a large number of this method, created a lot of unique Chinese terminology. For example, Chinese medicine early put the "five internal organs" than in the "five elements", so the five elements of wood, fire, soil, gold, water, respectively, represents the five internal organs of the liver, heart, spleen, lung.

(2) Literariness

Many of the classic works of Chinese medicine written in the form of classical Chinese, so the language has a strong characteristics of classical literature. With the passage of time, although its language has changed, but the exaggeration and exaggeration of the literature is still reflected in the literature of traditional Chinese medicine. For example, when it comes to disease prevention, commonly used "nip in the bud", "rainy day", describe the effect when used "miaoshouhuichun". These words are exaggerated and subjective, which are obviously different from modern medical rigorous and objective terms of science and technology.

(3)Relevance

Traditional Chinese medical terms are seemingly ordinary, but they are profound in meaning and often polysemous. Only by defining the context can the word meaning be accurately determined. For example, the word "phlegm fan" is a commonly used term in traditional Chinese medicine, and there are many causes. According to the different context and symptoms, it is divided into different situations.

(4)Philosophical speculative

Chinese medicine is based on the philosophy of philosophy based on the subject. From the perspective of philosophy to explore life, explore life, the day, the earth and the perfect combination of people into one, which contains a rich philosophical thinking. These theories are widely used in traditional Chinese medicine (TCM), and the doctrine of "phase with each other" contains simple dialectical materialism, such as "wood fire, fire, soil, water, fire ". And these relationships are not absolute. If there is "anti-grams" situation, then called "insult."

\section{Network Assisted Translation Strategy of Classical Chinese Medicine}

Internet Background. With the explosive pace of development, the Internet has rapidly become an important part of economy, society, culture and people's lives in just a few decades. The tentacles of the network economy from all aspects of the industry, a variety of online companies have emerged. As an important part of online enterprises, online tourism attracts everyone's attention with the new face, and the accompanying is the emergence and rapid development of online travel enterprises [7]. However, the development of network economy brings new opportunities for development and broad development stage for enterprises, and brings new methods and challenges to the translation of traditional Chinese medicine classics.

In recent years, although China's economic development rate has slowed down, but still higher than the average level of world economic development, the scale of Internet development has become increasingly strong, more and more residents began to use the internet. According to China internet development statistics report, the current internet users have increased to 849 million people, the annual growth rate of $4.7 \%$. At present, the number of Internet users in China has surpassed the United States, ranking first in the world. It can be seen that the Internet era has arrived, China's Internet industry has great vitality and vitality $[8,9]$. The rapid growth of the number of websites is also one of the important manifestations and driving factors of the development of China's internet. Blog, e-commerce and many other network applications demand surge, the increase in the number of domain names and create a simple operation of the website and other factors together, making the number of websites soared. According to the statistics report of China's internet development, up to now, the number of Chinese websites has reached 5 million 350 thousand, with an increase of 150 thousand in the same period, with an increase rate of $5.2 \%$. The translation of classical Chinese medical works should use the Internet 
platform, follow the principles and skills of translation to achieve the intellectualization, rapidity and accuracy of translation, as shown in figure 1.

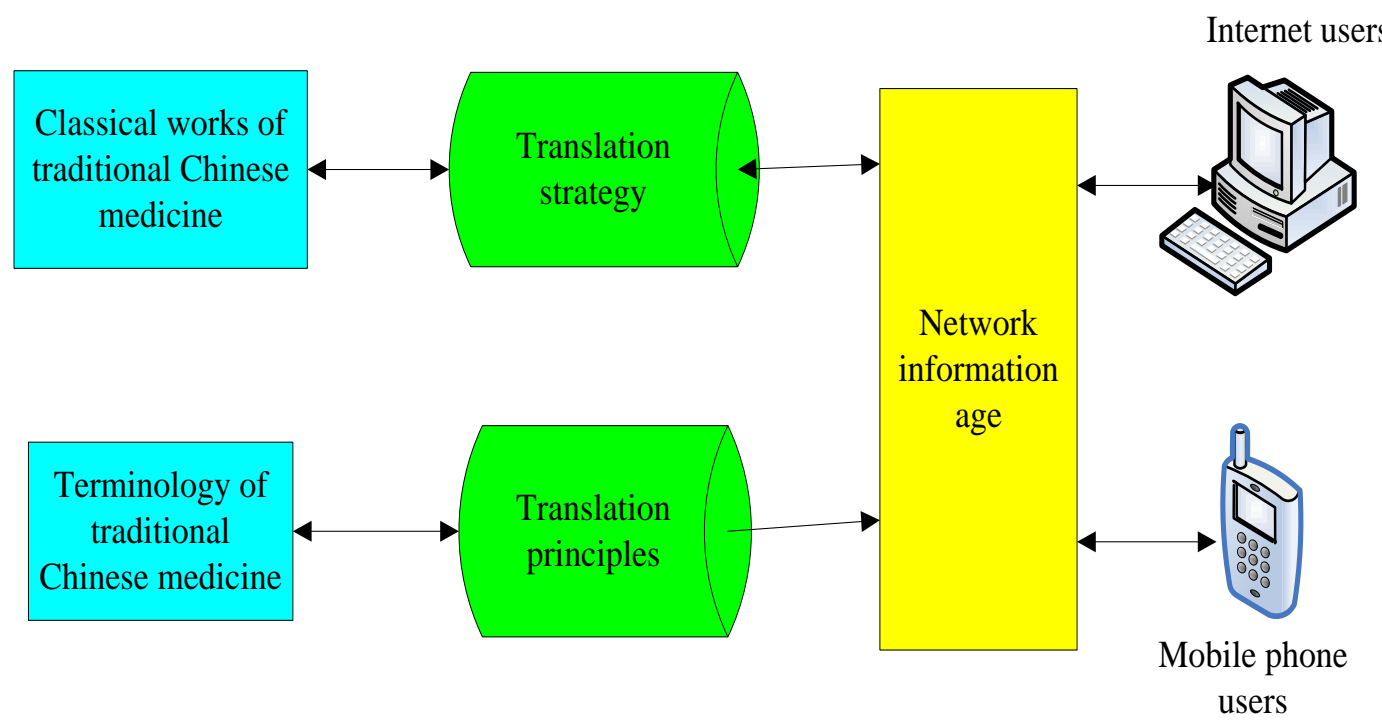

Figure 1. Network translation skills

Translation Principles.The principles that should be followed in the translation of traditional Chinese medicine are as follows, as shown in fig 2.

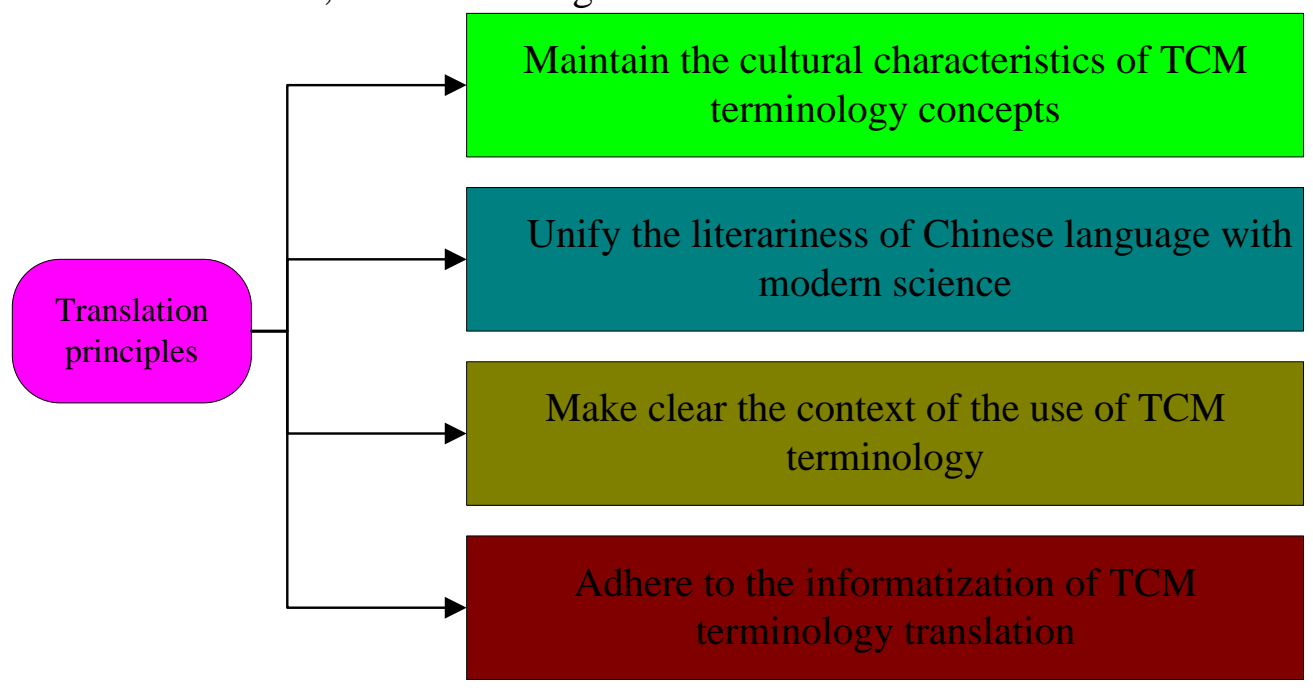

Figure 2. Principles to be followed in translation of TCM

(1) Maintain the cultural characteristics of TCM terminology concepts

Traditional Chinese medicine and modern western medicine are two different sciences, which are influenced by their respective national cultures and have a profound national cultural brand. Both the basic theory and the diagnosis and treatment are different, and the understanding of human physiology and pathology is also different. For example, traditional Chinese medicine emphasizes the whole concept in the medical treatment, pays attention to the unity of heaven and man; attaches importance to the medicinal taste and meridian tropism of drugs. But western medicine starts from the partial, the medicine aspect is mainly to eliminate the local lesion primarily [10]. In the process of translation, we should take into account the typical characteristics of Chinese culture, and reproduce the connotation of the original text as accurately as possible.

(2) Unify the literariness of Chinese language with modern science

Most of the classics of Chinese medicine are written in the form of ancient Chinese, and some of them are too literal. The translation should pay attention to Chinese language features from literary color is thick, the application of modern scientific system, making the translation from ancient natural philosophy of discussion into the scientific system of modern language to express the structure make the target language with English language. 
(3) Make clear the context of the use of TCM terminology

The semantics of TCM terms is abundant, and polysemy is very common. Therefore, we must make clear the context of its specific use. Grasp the meaning of term in context. Some esoteric Chinese medical terms do not have to be faithful to the source text and hard to translate. We can adjust the original text according to the context, change the equivalence with the original form, and pay attention to the functional equivalence.

(4) Adhere to the informatization of TCM terminology translation

Translation is the exchange of information across language and across cultures. The terms and concepts with strong philosophical speculation in Chinese medicine are the most difficult to understand and the most difficult to translate, but they are just the most part of cultural characteristics. The translation of TCM terms should emphasize the accuracy and completeness of information transfer, and the loss of accurate information concepts and content is less likely to be understood by the target readers. The translation is not excessive, the value-added information; nor less heat, so that the information cannot make depreciation; paradox, information distortion. And appropriately convey the information provided by the original text of traditional Chinese medicine.

\section{Translation Strategy}

Chinese medicine culture is broad and profound, rich in etymology. The choice of translation strategies is each one according to his lights. If we pay too much attention to the cultural connotation and literal meaning in the translation, we can hardly convey the medical connotation in the target language. If only according to the corresponding translation in terms of TCM medical significance of western medicine, Chinese medicine will make the culture eclipsed. At the same time, there are many terms and drugs in traditional Chinese medicine which are not found in western medicine, which increases the difficulty of translation. Skopos theory holds that the purpose of translation is to achieve the communicative function of target text in target language and to be accepted by target language readers $[11,12]$. Chinese medicine translation is to pass the ancient knowledge of traditional Chinese medicine to the world, let foreign readers understand the rich connotation of Chinese medicine, so that foreign medical practitioners understand their medical principles. Therefore, in translation, we should seek common features of Chinese and western medicine in the objective, accurate and scientific principles, and pass on the essence of TCM to the target language readers [13]. At the same time, we should maintain the traditional culture color of TCM. Different categories of TCM terms, etymology is different, so in the translation should be based on the communicative purpose, the choice of different translation strategies, flexible response.

Although traditional Chinese medicine and western medicine belong to two different medical systems, their treatment objects are the same, and there are some common things between them. Some Chinese medical terms can also find corresponding words in western medicine. For the translation of these terms, literal translation is not only convenient for translation, but also easy to be understood by foreign readers, such as psoriasis, menstrual blood, convulsions and other terms, in western medicine there are corresponding words.

\section{Summary}

With the rapid improvement of China's comprehensive strength and the extensive development of science and technology and cultural exchanges all over the world, the translation of TCM classics plays a more and more important role in the international communication of traditional Chinese medicine. However, due to the unique linguistic and cultural features of Chinese classics, the translation of traditional Chinese medicine has brought challenges to translation work. In the actual translation work, it is often impossible to achieve the complete equivalence effect between the translation and the original in terms of meaning, style and form. The equivalence between the translated works and the original in terms of meaning is at the core of the translation effect. If we can guarantee the equivalence of the original meaning of the translated text and the original text, the equivalent translation of language style, 
phonology, emotion and culture will be the best effect. Therefore, in the translation of Chinese classics, the translator should be good at using the network tools and translation skills and methods, so as to improve the quality of translation, and promote the international communication and dissemination of traditional Chinese medicine.

\section{Acknowlegement}

This work is supported by the shaanxi province project of social science fund: Research on the translation strategies of the names of traditional Chinese medicine classical works (No. 2016K012).

\section{References}

[1] Zheng L. [Research on Translation Strategies of Chinese Medicine in Traditional Cultural Context][J]. Zhongguo Zhong XI Yi Jie He Za Zhi, 2015, 2(10):1270-1273.

[2] Wang Y. On the Translation Strategies of Chinese Neologisms-A Case Study on Chinese Neologisms in 2015[J]. 2016(11):173-174.

[3] Zeng Q, Dai Z L. Analysis on the Translation Strategies of Chinese Characteristic Words in Mao Zedong's Poetry from the Perspective of Ecology[J]. Journal of Hunan First Normal University, 2017.

[4] Ming-Yang J I, Department F L. Studies on the Translation Strategies of Chinese Political Terms from the Perspective of Memetics[J]. Journal of Hainan Radio \& Tv University, 2016.

[5] Liu A, Wenjing D U, Tong X. Study on the Translation of Traditional Chinese Medicine Language from the Perspective of Pragmatics[J]. Western Journal of Traditional Chinese Medicine, 2017.

[6] Bai Y, Tao B C, Zhang B. Study on Translation Strategies of Chinese Medical Classics Reduplications from Translation Aesthetics Perspective[J]. Chinese Journal of Basic Medicine in Traditional Chinese Medicine, 2016.

[7] Wang N, Xue J M, Wang Z M. A Brief Study on Metaphor Translation Strategies in "Yellow Emperor's Canon of Internal Medicine" from the Perspective of Conceptual Metaphor[J]. Guiding Journal of Traditional Chinese Medicine \& Pharmacy, 2016.

[8] Ran Y, Yuan L U, Yao X. On Culture-Loaded Words in TCM and the Related Translation Strategies[J]. Western Journal of Traditional Chinese Medicine, 2015.

[9] Zuo B. Based on FCE CMI the Choice of Translation Strategies[C]// International Conference on Intelligent Transportation, Big Data and Smart City. IEEE, 2016:144-147.

[10] Li-Lan D U, Liu A J, Chen Z F, et al. Research status and analysis of English translation of traditional Chinese medicine terminologies between 2000 and 2012[J]. Education of Chinese Medicine, 2015.

[11]Lin Z Y. An Overview of Translation Studies of the Traditional Chinese Medicine into English from the Corpus-based Perspective[J]. Journal of Shanxi Datong University, 2016.

[12]Zuo B. The Standardization of the Translation of "Action and Indications" in the Traditional Chinese Patent Medicine Instruction[C]// International Conference on Education, Management and Computing Technology. 2015.

[13] Cao L, Zhang M. Analysis of Metaphorical Translation and the Characteristics of Traditional Chinese Medicine Language[J]. Western Journal of Traditional Chinese Medicine, 2016. 